\title{
WHEN THE NONTRIVIAL, SMALL DIVISORS OF A NATURAL NUMBER ARE IN ARITHMETIC PROGRESSION
}

\author{
HÙNG VIÊTT CHU
}

\begin{abstract}
Iannucci considered the positive divisors of a natural number $n$ that do not exceed $\sqrt{n}$ and found all forms of numbers whose such divisors are in arithmetic progression. In this paper, we generalize Iannucci's result by excluding the trivial divisors 1 and $\sqrt{n}$ (when $n$ is a square). Surprisingly, the length of our arithmetic progression cannot exceed 5 .
\end{abstract}

\section{INTRODUCTION AND MAIN RESULTS}

For each $n \in \mathbb{Z}_{\geq 1}$, its divisors not exceeding $\sqrt{n}$ are called small. The phrase small divisors, as used here, is not to be confused with classical small divisor problems of mathematical physics. In a recent paper, Iannucci [4] showed a nice and surprising result that charaterizes all natural numbers whose small divisors are in arithmetic progression (AP). In particular, Iannucci defined

$$
S_{n}:=\{d: d \mid n, d \leq \sqrt{n}\} .
$$

and analyzed the divisor-counting function to argue about the prime factorization of $n$ when $\left|S_{n}\right| \leq 6$. Then he showed that if $S_{n}$ is in AP, $\left|S_{n}\right|$ cannot be greater than 6 and finished the proof. For previous work on divisors in AP, see [1,7] and on small divisors, see [2, 5].

By Definition (1.1), 1 is in $S_{n}$. This trivial divisor gives information about the AP and plays a crucial role in the argument of Iannucci. Our goal is to exclude 1 and $\sqrt{n}$ from consideration to produce a more general theorem. For a natural number $n$, define

$$
A_{n}:=\{d: d \mid n, 1<d<\sqrt{n}\} .
$$

We shall determine all $n$ such that $A_{n}$ is in AP; that is,

$$
A_{n}=\{d, a+d, 2 a+d, \ldots,(k-1) a+d\},
$$

where $d$ is the first term, $a>0$ is the common difference and $k$ is the number of terms in the AP. Then the $i$ th term in $A_{n}$ is $d+(i-1) a$. Observe that if we write $n=p_{1}^{a_{1}} \cdots p_{\ell}^{a_{\ell}}$, where $p_{1}<p_{2}<\cdots<p_{\ell}$ are primes and $a_{i} \geq 1$, then $d=p_{1}$. For easy reading, we stick with these notation throughout the paper. By hypothesis, $A_{n}$ is in AP and $n \geq 2$. The following is our main theorem.

Theorem 1.1. For all $n \in \mathbb{Z}_{\geq 1}$, we have $\left|A_{n}\right| \leq 5$. In addition, if we let $p$, $q$, and $r$ denote distinct prime numbers, then one of the following is true:

(i) $n=p$ or $n=p^{2}$ for some $p$, hence $A_{n}=\emptyset$.

(ii) $n=p q$ for some $p<q$, hence $A_{n}=\{p\}$.

2010 Mathematics Subject Classification. Primary: 11B25 .

Key words: divisor, arithmetic progression. 
(iii) $n=p^{3}$ or $n=p^{4}$ for some $p$, hence $A_{n}=\{p\}$.

(iv) $n=p^{5}$ for some $p$, hence $A_{n}=\left\{p, p^{2}\right\}$.

(v) $n=p q^{2}$, where $p<q$, hence $A_{n}=\{p, q\}$.

(vi) $n=p^{2} q$, where $p^{2}<q$, hence $A_{n}=\left\{p, p^{2}\right\}$.

(vii) $n=p^{2} q$, where $p<q<p^{2}$, hence $A_{n}=\{p, q\}$.

(viii) $n=p^{6}$ for some $p$, hence $A_{n}=\left\{p, p^{2}\right\}$.

(ix) $n=36$, hence $A_{n}=\{2,3,4\}$.

(x) $n=p q r$, where $p<q<r$ such that $2 q=p+r$, hence $A_{n}=\{p, q, r\}$. (We have infinitely many triple of primes $(p, q, r)$ such that $2 q=p+r$ because there are arbitrarily long arithmetic progressions of primes [6].)

(xi) $n=24$, hence $A_{n}=\{2,3,4\}$.

(xii) $n=60$, hence $A_{n}=\{2,3,4,5,6\}$.

Since $A_{n} \subset S_{n}$ for any $n \in \mathbb{Z}_{\geq 1}$, it is straightforward to deduce [4, Theorem 4] from our Theorem 1.1. As usual, we have the divisor-counting function

$$
\tau(n):=\sum_{d \mid n} 1
$$

Since $\tau(n)$ is multiplicative, for the $m$ distinct primes $p_{1}<p_{2}<\cdots<p_{m}$, and natural numbers $a_{1}, a_{2}, \ldots, a_{m}$,

$$
\tau\left(p_{1}^{a_{1}} p_{2}^{a_{2}} \cdots p_{m}^{a_{m}}\right)=\left(a_{1}+1\right)\left(a_{2}+1\right) \cdots\left(a_{m}+1\right) .
$$

If $n=b c$ and $b \leq c$, then $b \leq \sqrt{n} \leq c$; hence,

$$
\tau(n)= \begin{cases}2\left|S_{n}\right|-1 & \text { if } n \text { is a square } \\ 2\left|S_{n}\right| & \text { if } n \text { is not a square. }\end{cases}
$$

Since

$$
\left|S_{n}\right|= \begin{cases}\left|A_{n}\right|+2 & \text { if } n \text { is a square } \\ \left|A_{n}\right|+1 & \text { if } n \text { is not a square }\end{cases}
$$

we have

$$
\tau(n)= \begin{cases}2\left|A_{n}\right|+3 & \text { if } n \text { is a square } \\ 2\left|A_{n}\right|+2 & \text { if } n \text { is not a square. }\end{cases}
$$

In Section 2, we prove that for all $n \in \mathbb{Z}_{\geq 1},\left|A_{n}\right| \leq 5$. In Section 2 and the Appendix, we analyze $\left|A_{n}\right|=k$ for each $0 \leq k \leq 5$ to prove Theorem 1.1. Since the case analysis when $k=5$ is lengthy and not interesting, we move it to the Appendix.

\section{Proof of the First PART OF THEOREM 1.1}

In this section, we prove that $\left|A_{n}\right| \leq 5$. Note that this bound is sharp since $A_{60}=$ $\{2,3,4,5,6\}$. We state an immediate consequence of [4, Lemma 2] that will be used in due course.

Lemma 2.1. If $(d, a)=(2,1)$ or $(3,2)$, then $\left|A_{n}\right| \leq 5$.

Lemma 2.2. If $a=1$ or $a=2$, then $\left|A_{n}\right|=k \leq 5$. 
Proof. For a contradiction, we assume that $\left|A_{n}\right| \geq 6$. If $n$ has exactly one prime divisor, then write $n=p^{u}$ for some prime $p$ and $u \geq 2 k+1$. So, $A_{n}=\left\{p, p^{2}, p^{3}, \ldots, p^{k}\right\}$. However, $p, p^{2}, p^{3}$ are not in AP for all $p$. Hence, $n$ must have at least two distinct prime divisors, called $p_{1}$ and $p_{2}$, where $p_{1}<p_{2}$.

(1) First, we consider $a=1$. The two smallest numbers in $A_{n}$ are $p_{1}$ and $p_{1}+1$. Since $p_{1}<p_{2}$, it follows that $p_{1}+1 \leq p_{2}$. There are two possibilities:

(i) If $p_{1}+1$ is not a prime, then $p_{1}+1 \geq p_{1}^{2}$ because $\left(p_{1}+1\right) \mid n$. For all primes $p$, we have $p^{2}>p+1$, so this case cannot happen.

(ii) If $p_{1}+1$ is a prime, then $p_{1}+1=p_{2}$. So, $p_{1}=2$ and $p_{2}=3$. It follows that $A_{n}=\{2,3,4, \ldots, m\}$ for $m \geq 7$. However, by Lemma 2.1, we have $m \leq 6$, a contradiction.

(2) Suppose that $a=2$. The three smallest numbers in $A_{n}$ are $p_{1}, p_{1}+2$ and $p_{1}+4$. Suppose that $n$ has three distinct prime factors. It follows that $p_{1}+2 \leq p_{2}$; otherwise, $\left(p_{1}, p_{2}\right)=(2,3)$, which, by Lemma 2.1, implies that $\left|A_{n}\right| \leq 5$, a contradiction. Since $p_{1}+2 \leq p_{2}$, we have $p_{1}+4 \leq p_{2}+2 \leq p_{3}$. There are two possibilities for $p_{1}+2$ :

(i) If $p_{1}+2$ is not a prime, then $p_{1}+2 \geq p_{1}^{2}$, which implies that $p_{1}=2$. Then the three smallest numbers in $A_{n}$ are $2,4,6$. However, $6 \mid n$ implies that $3 \mid n$, so $3 \in A_{n}$, a contradiction.

(ii) If $p_{1}+2$ is a prime, then $p_{1}+2=p_{2}$. We consider two subcases.

(a) If $p_{1}+4=p_{3}$, then at least one of $p_{1}, p_{2}, p_{3}$ is divisible by 3 . It must be that $p_{1}=3$. So, $A_{n}=\{3,5,7, \ldots, 2 m-1\}$ for some $m \geq 7$. By Lemma 2.1, we have a contradiction.

(b) If $p_{1}+4<p_{3}$, Write $p_{1}+4=p_{1}^{u_{1}} p_{2}^{u_{2}}$ for some $u_{1}, u_{2} \geq 0$. Then

$$
\begin{aligned}
p_{1}^{u_{1}} p_{2}^{u_{2}}-2 & =p_{1}+2=p_{2}, \text { and so } \\
p_{1}^{u_{1}} p_{2}^{u_{2}} & =p_{2}+2 .
\end{aligned}
$$

Clearly, either $u_{1}$ or $u_{2}=0$. If $u_{1}=0, p_{2}^{u_{2}}=p_{2}+2$ has no odd prime solutions $p_{2}$ for all $u_{2} \geq 0$. If $u_{2}=0, p_{1}^{u_{1}}=p_{2}+2=p_{1}+4$ has no prime solutions $p_{1}$ for all $u_{1} \geq 0$.

So, $n$ has exactly two distinct prime factors $p_{1}$ and $p_{2}$. We write $p_{1}+4=p_{1}^{u_{1}} p_{2}^{u_{2}}$ for some $u_{1}, u_{2} \geq 0$. As above, we have a contradiction.

Therefore, $k=\left|A_{n}\right| \leq 5$, as desired.

Lemma 2.3. If $k:=\left|A_{n}\right| \geq 6$, then $a$ is even and $p_{1} \geq 3$, where $a$ is the common difference and $p_{1}$ is the least term in $A_{n}$.

Proof. Due to the proof of Lemma 2.2, $n$ has at least one prime factor other than $p_{1}$. Let $p_{2}$ denote the prime factor. We consider two cases corresponding to whether $p_{1} \geq 3$ or $p_{1}=2$.

(1) Assume $p_{1} \geq 3$. Since $p_{1}+a \leq p_{2}$, we have either $p_{1}+a=p_{2}$ (if $p_{1}+a$ is a prime) or $p_{1}+a=p_{1}^{2}$ (if $p_{1}+a$ is not a prime). In both cases, $a$ is even.

(2) Assume $p_{1}=2$. Then $A_{n}=\{2,2+a, 2+2 a, \ldots, 2+(k-1) a\}$. We show a contradiction. The third term in $A_{n}$ is $2(a+1)=p_{1}(a+1)$. Hence, we have $a+1 \mid n$ and $a+1<a+2$, so $a+1=2$ and $a=1$. By Lemma 2.2 $k \leq 5$, a contradiction. 
We have shown that if $\left|A_{n}\right|=k \geq 6, a$ must be even and $p_{1} \geq 3$.

Lemma 2.4. If $a>2$ and $k \geq 6$, then $k \geq a+3$.

Proof. Suppose otherwise. That is, $a>2$ and $k \geq 6$, but $k \leq a+2$. We will show a contradiction after proving the two following statements (i) and (ii).

(i) $p_{1} \nmid a$.

(ii) $n$ has at least $k-\lfloor(k-1) / 3\rfloor$ distinct prime factors.

Proof of (i): Suppose that $p_{1} \mid a$. Write $a=u p_{1}$ for some $u \in \mathbb{Z}_{\geq 1}$. Then

$$
A_{n}=\left\{p_{1},(u+1) p_{1},(2 u+1) p_{1}, \ldots,((k-1) u+1) p_{1}\right\} .
$$

Hence, $u+1$ and $2 u+1 \in A_{n}$. Since $u+1<(u+1) p_{1}$, we have $u+1=p_{1}$. Furthermore, since $u+1<2 u+1$, we have $2 u+1 \geq(u+1) p_{1}$, hence $p_{1} \leq 1$, which contradicts the primeness of $p_{1}$. This contradiction tells us that the assumption $p_{1} \mid a$ is false. So, $p_{1}$ does not divide $a$.

Proof of (ii): Define

$$
S:=\left\{p_{1}+j a: 1 \leq j \leq k-1\right\} \subset A_{n},
$$

$S_{1}:=\left\{s \in S: p_{1} \mid s\right\}$, and $S_{2}:=S \backslash S_{1}$. Due to claim (i), $p_{1} \mid s$ for some $s \in S$ if and only if $s=p_{1}+j a$ for some $1 \leq j \leq k-1$ and $p_{1}$ divides $j$. So, $\left|S_{1}\right|=\left\lfloor(k-1) / p_{1}\right\rfloor \leq$ $\lfloor(k-1) / 3\rfloor$ (due to Lemma 2.3) and so, we have

$$
\left|S_{2}\right|=|S|-\left|S_{1}\right| \geq(k-1)-\lfloor(k-1) / 3\rfloor .
$$

We claim that $s$ is prime for all $s \in S_{2}$. Otherwise, if $s=j a+p_{1} \in S_{2}$ is composite, then there exist $1 \leq b, c<j$ such that $j a+p_{1}=\left(b a+p_{1}\right)\left(c a+p_{1}\right)$. Note that $b, c \geq 1$ because $p_{1} \nmid s$ if $s \in S_{2}$. We have

$$
\begin{aligned}
(k-1) a+p_{1} & \geq j a+p_{1}=\left(b a+p_{1}\right)\left(c a+p_{1}\right) \\
& \geq\left(a+p_{1}\right)^{2}=\left(a+2 p_{1}\right) a+p_{1}^{2}>(k-1) a+p_{1},
\end{aligned}
$$

a contradiction. The last inequality is due to the assumption that $a+2 \geq k$. Therefore, all numbers in $S_{2}$ are prime; together with $p_{1}$, we know that $n$ has at least $k-\lfloor(k-1) / 3\rfloor$ distinct prime factors. This completes the proof of statement (ii).

We now deduce a contradiction, which rejects our supposition that $k \leq a+2$. Due to statement (ii), we have $\tau(n) \geq 2^{k-\lfloor(k-1) / 3\rfloor}$. By (1.3), we obtain

$$
k=\left|A_{n}\right| \geq \frac{\tau(n)-3}{2} \geq \frac{2^{k-\lfloor(k-1) / 3\rfloor}-3}{2},
$$

which contradicts our assumption that $k \geq 6$. This contradiction shows $k \geq a+3$.

Lemma 2.5. If $a>2$, then $\left|A_{n}\right|=k \leq 5$.

Proof. Assume, by contradiction, that $a>2$ and $k \geq 6$. By Lemma 2.3, $a$ is even; hence, $a \geq 4$. By Bertrand's postulate, there exists a prime $q$ such that $a / 2<q<a$. Let

$$
Q=\left\{p_{1}+a, p_{1}+2 a, \ldots, p_{1}+q a\right\} .
$$

Since $q<a$, by Lemma2.4, $Q \subset A_{n}$. Since $q \nmid a$, it follows that no two elements of $Q$ are congruent modulo $q$. Hence, there exists $j$ such that $1 \leq j \leq q$ such that $q \mid j a+p_{1}$. Therefore, $q \mid n$ and $q<\sqrt{n}$. So, $q \in A_{n}$. The only possibility is $q=p_{1}$ because $q<a$. 
WHEN THE NONTRIVIAL, SMALL DIVISORS OF A NATURAL NUMBER ARE IN ARITHMETIC PROGRESSIO\$

Observe that $p_{1}+q a=q(a+1)$, so $a+1 \in A_{n}$. Write

$$
A_{n}=\{q, q+a, q+2 a, \ldots, q+(k-1) a\}
$$

to see that $a+1 \notin A_{n}$. We have a contradiction. Therefore, $k \leq 5$.

Proof of the first part of Theorem 1.1 The claim that $\left|A_{n}\right| \leq 5$ follows immediately from Lemma 2.2 and Lemma 2.5 .

\section{PROOFS OF THE SECOND PART OF THEOREM 1.1}

We consider natural numbers $n$ such that $\left|A_{n}\right|$ does not exceed 5 and $A_{n}$ is in AP. We prove by case analysis with each case corresponding to each $0 \leq k \leq 4$. For each value of $k$, we may itemize further if necessary. For $k=5$, see the Appendix.

Proof of the second part of Theorem 1.1 We consider 5 cases.

(1) Case 1: $k=0$. By (1.3), $\tau(n)=2$ or 3. By (1.2), $n=p$ or $n=p^{2}$ for some prime $p$. In both cases, $A_{n}=\emptyset$, which is vacuously in AP. This corresponds to item (i) of Theorem 1.1 .

(2) Case 2: $k=1$. By (1.3), $\tau(n)=4$ or 5. By (1.2), $n=p q, p^{3}$ or $p^{4}$ for some primes $p<q$. In all cases, $A_{n}=\{p\}$, which is vacuously in AP. This corresponds to items (ii) and (iii).

(3) Case 3: $k=2$. By (1.3), $\tau(n)=6$ or 7. By (1.2), $n=p^{5}, p q^{2}, p^{2} q$ or $p^{6}$ for some primes $p<q$.

(a) If $n=p^{5}$, then $A_{n}=\left\{p, p^{2}\right\}$, which is in AP. This is item (iv).

(b) If $n=p q^{2}$, then $A_{n}=\{p, q\}$, which is in AP. This is item (v).

(c) If $n=p^{2} q$, then we have two subcases.

(i) If $p^{2}<q$, then $A_{n}=\left\{p, p^{2}\right\}$. This is item (vi).

(ii) If $p^{2}>q$, then $A_{n}=\{p, q\}$. This is item (vii).

(d) If $n=p^{6}$, then $A_{n}=\left\{p, p^{2}\right\}$, which is in AP. This is item (viii).

(4) Case 4: $k=3$. By (1.3), $\tau(n)=8$ or 9 . By (1.2), $n=p q r, p q^{3}, p^{3} q, p^{7}, p^{2} q^{2}$ or $p^{8}$ for some primes $p<q<r$.

(a) If $n=p^{7}$ or $p^{8}$, because $p, p^{2}, p^{3}$ are not in AP, we eliminate this case.

(b) If $n=p^{2} q^{2}$, then $A_{n}=\left\{p, p^{2}, q\right\}$.

(i) If $q>p^{2}$, then in order that $A_{n}$ is in AP, $q=2 p^{2}-p=p(2 p-1)$, which contradicts that $q$ is a prime. We eliminate this case.

(ii) If $p<q<p^{2}$, then $2 q=p^{2}+p=p(p+1)$. If $p \geq 3$, then $q=p \frac{p+1}{2}$ is not a prime because $\frac{p+1}{2} \geq 2$. Hence, $p=2, q=3$, and $n=36$. This is item (ix).

(c) If $n=p q r$, either $r>p q$ or $r<p q$.

(i) If $r>p q$, then $A_{n}=\{p, q, p q\}$. Since $A_{n}$ is in AP, we have $p=$ $2 q /(q+1)<2$, a contradiction.

(ii) If $r<p q$, then $A_{n}=\{p, q, r\}$. Then $2 q=p+r$. This is item (x).

(d) If $n=p q^{3}$, then $A_{n}=\{p, q, p q\}$. So, $2 q=p+p q$ and so, $p=2 q /(q+1)<$ 2 , a contradiction.

(e) If $n=p^{3} q$, either $q>p^{3}$ or $q<p^{3}$.

(i) If $q>p^{3}$, then $A_{n}=\left\{p, p^{2}, p^{3}\right\}$, which is not in AP. 
(ii) If $q<p^{3}$, then $A_{n}=\left\{p, p^{2}, q\right\}$. If $q>p^{2}$, then $A_{n}$ is in AP if and only if $2 p^{2}=p+q$. So, $q=p(2 p-1)$, which contradicts that $q$ is a prime. If $q<p^{2}$, then $A_{n}$ is in AP if and only if $2 q=p+p^{2}=$ $p(p+1)$. Equivalently, $q=p \frac{p+1}{2}$. In order that $q$ is prime, we must have $p=2, q=3$, and $n=24$. This is item (xi).

(5) Case 5: $k=4$. By (1.3), $\tau(n)=10$ or 11 . By (1.2), $n=p^{9}, p q^{4}, p^{4} q$ or $p^{10}$ for some primes $p<q$.

(a) If $n=p^{9}$ or $p^{10}$, then $A_{n}$ is not in AP because $p, p^{2}, p^{3}$ are not in AP. We eliminate this case.

(b) If $n=p q^{4}$, then $A_{n}=\left\{p, q, p q, q^{2}\right\}$. It follows that $q+p q=p+q^{2}$. So, $p=q$, which contradicts our assumption that $p<q$.

(c) If $n=p^{4} q$, then we consider three subcases.

(i) If $p<q<p^{2}$, then $A_{n}=\left\{p, q, p^{2}, p q\right\}$. In order that $A_{n}$ is in AP, $q+p q=p^{2}+p$ and so, $p=q$, a contradiction.

(ii) If $p^{2}<q<p^{4}$, then $A_{n}=\left\{p, p^{2}, p^{3}, q\right\}$. In order that $A_{n}$ is in AP, the three numbers $p, p^{2}$, and $p^{3}$ cannot be the three smallest elements of $A_{n}$ because $\left\{p, p^{2} p^{3}\right\}$ are not in AP. Hence, $p^{2}<q<p^{3}$. We have $p^{3}+p=p^{2}+q$. So, $p \mid q$, a contradiction.

(iii) If $p^{4}<q$, then $A_{n}=\left\{p, p^{2}, p^{3}, p^{4}\right\}$, which is not in AP.

We have analyzed all cases up to $k=4$. For $k=5$, see the Appendix.

\section{ACKNOWLEDGEMENT}

The author would like to thank the anonymous referees for helpful suggestions that improve the exposition of this paper.

\section{APPENDIX A. When $\left|A_{n}\right|=5$}

By (1.3), $\tau(n)=12$ or 13 . By (1.2), $n=p q^{5}, p^{5} q, p^{2} q^{3}, p^{3} q^{2}, p^{2} q r, p q^{2} r$ or $p q r^{2}$ for some primes $p<q<r$. We can eliminate the cases $n=p^{11}$ and $n=p^{12}$ because $\left\{p, p^{2}, p^{3}\right\}$ are not in AP.

(1) If $n=p q^{5}$, then $A_{n}=\left\{p, q, p q, q^{2}, p q^{2}\right\}$ with $p<q<p q<q^{2}<p q^{2}$. So, $p+p q=2 q$ and so $p=2 q /(q+1)<2$, a contradiction.

(2) If $n=p^{5} q$, we consider the following subcases.

(a) If $q<p^{3}$, then $A_{n}=\left\{p, p^{2}, p^{3}, q, p q\right\}$.

(i) If $q<p^{2}$, then $p<q<p^{2}<p q<p^{3}$. So, $2 q=p+p^{2}=p(p+1)$, which gives $(p, q)=(2,3)$. However, $A_{96}$ is not in AP.

(ii) If $p^{2}<q<p^{3}$, then $p<p^{2}<q<p^{3}<p q$. So, $2 q=p^{2}+p^{3}=$ $p^{2}(p+1)$, which implies that $p \mid q$, which contradicts that $q$ is a prime greater than $p$.

(b) If $p^{3}<q$, then the three smallest numbers in $A_{n}$ are $p, p^{2}, p^{3}$, which are not in AP.

(3) If $n=p^{2} q^{3}$, either $p^{2}>q$ or $p^{2}<q$.

(a) If $p<q<p^{2}$, then $A_{n}=\left\{p, q, p^{2}, p q, q^{2}\right\}$ with $p<q<p^{2}<p q<q^{2}$. So, $2 p q=p^{2}+q^{2}$, which implies that $p(2 q-p)=q^{2}$ and so, $p \mid q$, a contradiction. 
(b) If $p^{2}<q$, then $A_{n}=\left\{p, p^{2}, q, p q, p^{2} q\right\}$ with $p<p^{2}<q<p q<p^{2} q$. So, $2 q=p^{2}+p q$; thus, $p=2 q /(p+q)<2$, a contradiction.

(4) If $n=p^{3} q^{2}$, either $p^{3}>q^{2}$ or $p^{3}<q^{2}$.

(a) If $p^{2}<q^{2}<p^{3}$, then $A_{n}=\left\{p, q, p^{2}, p q, q^{2}\right\}$ with $p<q<p^{2}<p q<q^{2}$. So, $2 p q=p^{2}+q^{2}$, which implies that $p \mid q$, a contradiction.

(b) If $p^{3}<q^{2}$, then $A_{n}=\left\{p, p^{2}, p^{3}, q, p q\right\}$.

(i) If $q<p^{2}$, we have $p<q<p^{2}<p q<p^{3}$. So, $2 p q=p^{3}+p^{2}$; equivalently, $q=p \frac{p+1}{2}$, which gives $(p, q)=(2,3)$. However, $A_{72}=$ $\{2,3,4,6\}$, which is not in AP.

(ii) If $p^{2}<q<p^{3}$, we have $p<p^{2}<q<p^{3}<p q$. So, $2 q=p^{2}+p^{3}=$ $p^{2}(p+1)$, which contradicts the primeness of $q$.

(iii) If $p^{3}<q$, we have $p<p^{2}<p^{3}<q<p q$. Since $p, p^{2}, p^{3}$ are not in AP, we eliminate this case.

(5) If $n=p^{2} q r$, either $p^{2} q>r$ or $p^{2} q<r$.

(a) If $p^{2} q>r$, then $A_{n}=\left\{p, p^{2}, q, p q, r\right\}$. If $q>p^{2}$, the three smallest numbers in $A_{n}$ are $p<p^{2}<q$. In order that $A_{n}$ is in AP, $2 p^{2}=p+q$ and so, $p \mid q$, a contradiction. So, $p<q<p^{2}$. We consider two further subcases $q<r<p^{2}$ and $p^{2}<r$.

(i) If $p<q<r<p^{2}<p q$, then $q+p q=p^{2}+r$ and $2 q=p+r$. Substituting $r=2 q-p$ into the former, we have $p q=p^{2}+q-p$, which implies that $p \mid q$, a contradiction.

(ii) If $p^{2}<r$, then the three smallest numbers are $p<q<p^{2}$. So, $2 q=p(p+1)$, which gives $(p, q)=(2,3)$.

$*$ If $r<p q$, then $r=5$ and $n=60$. This is item (xii).

$*$ If $r>p q$, then $p<q<p^{2}<p q<r$. So, $2 p q=p^{2}+r$ and so, $r=8$, which contradicts that $r$ is prime.

(b) If $p^{2} q<r$, then $A_{n}=\left\{p, p^{2}, q, p q, p^{2} q\right\}$. Similar as above, we have $p<$ $q<p^{2}$. So, $p<q<p^{2}<p q<p^{2} q$. Since the three smallest numbers are $p<q<p^{2}$, we have $2 q=p+p^{2}$, which gives $(p, q)=(2,3)$. However, $2,3,4,6,12$ are not in AP.

(6) If $n=p q^{2} r$, we consider two cases $r>p q$ and $r<p q$.

(a) If $r>p q$, then the three smallest numbers in $A_{n}$ are $p<q<p q$. So, $2 q=p+p q$, which gives $p=2 q /(q+1)<2$, a contradiction.

(b) If $r<p q$, then the four smallest numbers in $A_{n}$ are $p<q<r<p q$. So, $2 r=p q+q=q(p+1)$, which implies that either $r \mid q$ or $r \mid(p+1)$. However, both cases contradict that $p<q<r$.

(7) If $n=p q r^{2}$, then $A_{n}=\{p, q, p q, r, p r\}$. Either $p<q<p q<r<p r$ or $p<q<r<p q<p r$. None of these cases (which we have seen above) gives us an $A_{n}$ in AP.

\section{REFERENCES}

[1] W. Banks, J. Friedlander, and F. Luca, Integers without divisors from a fixed arithmetic progression, Forum Math. 20 (2008), 1005-1037.

[2] D. Bruijn, N. Tengbergen, and D. Kruyswijk, On the set of divisors of a number, Nieuw Arch. Wiskd. 23 (1951), 191-193. 
[3] K. Ford, F. Luca, C. Pomerance, and J. Shallit, On the parity of the number of small divisors of $n$, in C. Pomerance and T. Rassias, eds., Analytic Number Theory. In Honor of Helmut Maier's 60th Birthday, Springer, 2015, pp. 93-100.

[4] D. E. Iannucci, When the small divisors of a natural number are in arithmetic progression, Integers (2018).

[5] D. E. Iannucci, On sums of the small divisors of a natural number, preprint (2019). Available at https://arxiv.org/abs/1910.11835.

[6] B. Green and T. Tao, The primes contain arbitrarily long arithmetic progressions, Ann. of Math. 167 (2008), 481-547.

[7] P. Varbanek and P. Zarzycki, Divisors of the Gaussian integers in an arithmetic progression, $J$. Number Theory 33 (1989), 152-169.

Email address: hungchu2@illinois.edu

Department of MATHEMATics, University of Illinois at Urbana-Champaign, URbana, IL 61820, USA 